Chapter 10

\title{
Evoked EMG Makes Measurement of Muscle Tone Possible by Analysis of the H/M Ratio
}

\author{
Satoru Kai and Koji Nakabayashi \\ Additional information is available at the end of the chapter \\ http://dx.doi.org/10.5772/55783
}

\section{Introduction}

\subsection{Evoked EMG - Mechanism of evoked EMG}

When we humans perform a physical activity, it is contraction of skeletal muscles that is responsible as its driving force. Active state of a skeletal muscle during motion can be viewed on an electromyogram (EMG). Because it is non-invasive and easy to handle, surface EMG has been widely used in the field of rehabilitation as in therapeutic exercise, training, motion analysis, and research.

When a peripheral nerve is subjected to percutaneous electrical stimulation, action potentials are induced in the innervated skeletal muscle. The induced action potential is recorded by evoked EMG, which includes the H-wave, the M-wave, and the F-wave (Fig. 1). The H-wave is a good indicator of the strength and distribution of the stimulus input from muscle spindle to the motor neuron pool, which lies at the site of the anterior horn of the spinal cord. The Hwave is commonly used, therefore, in the diagnosis of peripheral neuropathy (Kaeser 1973). The H-wave is also used to examine the state of muscle tone and spasticity, or other movement disorders of the central nervous system.

\subsubsection{H-wave (H-reflex)}

The name H-wave was derived from that of Johann Hoffmann, who found the response for the first time in 1918. Weak electrical stimulation can excite group Ia fibers from muscle spindle, and the antidromic impulse gets conducted to the spinal cord. Afferent fibers connect, via a synapse, with the alpha motor neuron in the spinal cord. Excitatory postsynaptic potential (EPSP) causes the excitement of the alpha motor neuron of the anterior horn cells in the spinal cord. The action potential that reaches from excitatory alpha motor neuron to skeletal muscle 
is called $\mathrm{H}$-wave or H-response. With soleus or flexor carpi radialis muscle the H-wave is observed at rest, but in other muscles the H-wave can only be induced with mild voluntary contraction.

\section{M-wave}
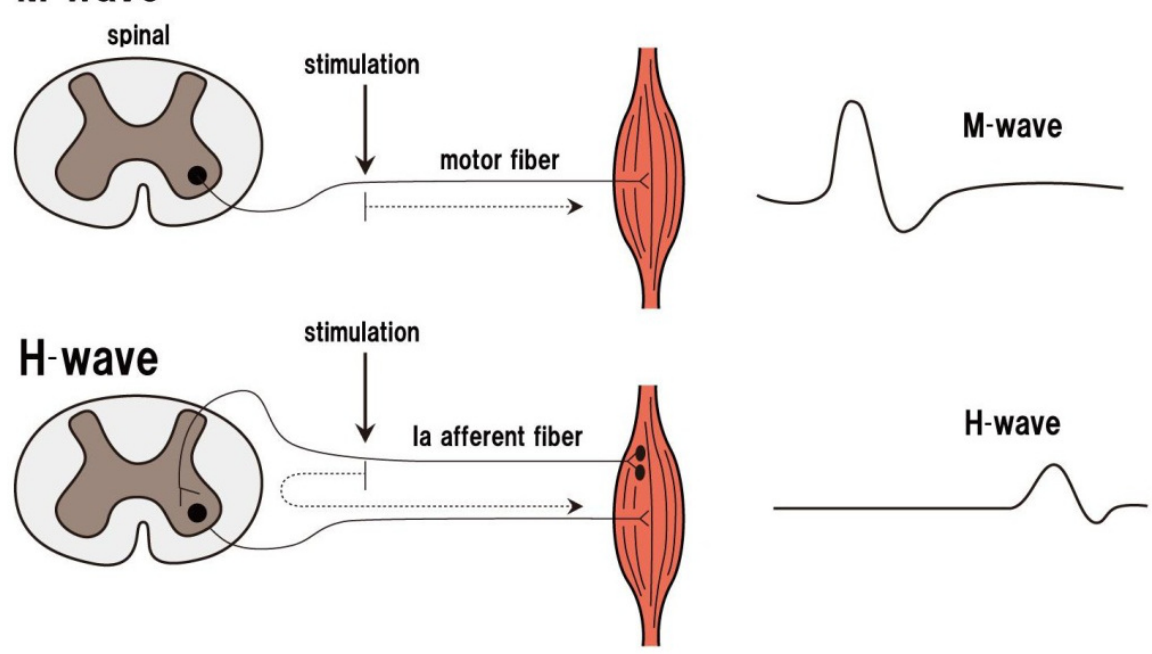

\section{F-wave}
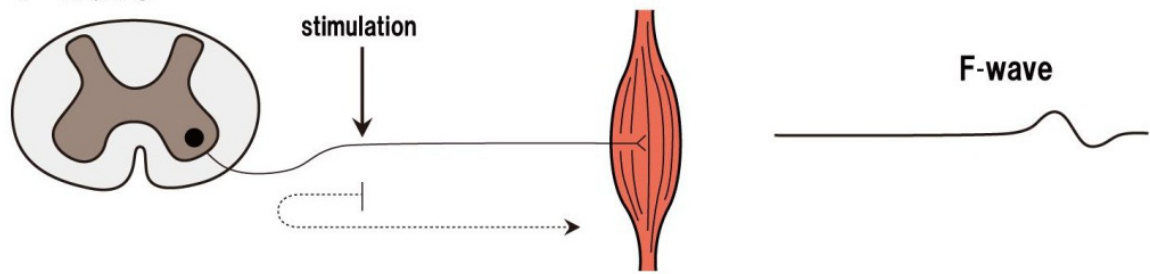

M-wave: Excitement is conducted to efferent. H-wave: Excitement is conducted via the monosynaptic reflex. F-wave: Excitement is conducted to antidromic.

Figure 1. Evoked EMG

\subsubsection{M-wave (M-response)}

Alpha motor neurons are activated directly by a gradual increase in the intensity of electrical stimulation, which occurs as the activity of Ia fiber is enhanced. The action potential of the skeletal muscle caused by this excitement is called M-wave or M-response.

\subsubsection{F-wave (F-response)}

If an alpha motor neuron receives a strong electrical stimulation, its antidromic impulse is conducted to axons of all motor neurons that have received the stimulus. The impulse is to 
reach the innervated skeletal muscle after re-firing at part of the axon hillock of anterior horn cells in the spinal cord. The action potential in question is called F-wave or F-response. The Fwave is said to be evoked on any peripheral muscle.

\subsection{Measurement of $\mathrm{H}-$ and $\mathrm{M}$-wave}

With evoked EMG, the action potential is derived from a skeletal muscle that is innervated by electrical stimulation conducted to peripheral nerves.

In this section, we briefly introduce the measurement method of $\mathrm{H}$-wave in soleus muscle in accordance with the guidelines of clinical neurophysiology test as stipulated by International Federation of Clinical Neurophysiology. Kaeser advocated that the measurement of H-wave be standardized in the study of spinal reflex (Kaeser 1973). It is not always easy to apply his method in clinical situations, but we recommend that you should examine his method carefully because it is considered to be a correct way of recording the H-wave.

\subsubsection{Postural position at measurement}

Because $\mathrm{H}$-wave is susceptible to the effects of posture, it is necessary to maintain the same posture during the measurement. A change in posture gives rise to changes in the length of muscles, which in turn bring on a change in the activity of muscle spindle receptors involved in the $\mathrm{H}$-wave. That is how postural change can cause variability in $\mathrm{H}$-wave.

When you try to evoke the $\mathrm{H}$-wave of soleus muscle, it is important to prevent soleus muscle, and gastrocnemius muscle, from stretching. For the preventive purpose, you instruct the subject to mildly flex the knee joint (about 30 degrees). And because the $\mathrm{H}$-wave of soleus muscle is inhibited by ankle dorsiflexion, the ankle joint is fixed at a mild plantar flexion position (about 20 degrees).

\subsubsection{Stimulus conditions}

The optimal duration of stimulation to elicit the $\mathrm{H}$-wave is $0.5 \mathrm{~ms}$ or $1.0 \mathrm{~ms}$; the choice is due to the difference in the strength-duration curves between axons of motor neurons and afferent group Ia fibers. Optimal conditions to excite group Ia fibers are 1) stimulation at a low enough intensity not to excite the axons of motor neurons, and 2) placement of the cathode of the stimulating electrode on the nerve, with the anode placed distal from the cathode on the run of the nerve. Because it is a reflex through a synapse, H-wave is easy to cause "habituation". That means that shorter stimulation intervals would tend to inhibit $\mathrm{H}$-wave due to the decreased synaptic connection in the spinal cord. It is therefore important that the interval of stimulations be long enough, but you must also remember that the subjects would sometimes be poised when the stimulation interval is more than 5 seconds. For removal of the inhibitive influence of habituation, it is desirable to set the interval for stimuli at 10 seconds or more.

Clinically, it is often convenient, and optimal, to start with a rate of $1 \mathrm{~Hz}$ for $\mathrm{H}$-wave measurement; the rate of stimulation is then decreased step by step (by $0.2-0.3 \mathrm{~Hz}$ ) so that latency and amplitude can be measured. 


\subsubsection{Recording method (Fig. 2)}

$\mathrm{H}$-wave recording is often done with surface EMG. The skin surface is wiped clean before attaching an electrode to keep electrical resistance as low as possible. To record the H-wave from soleus muscle selectively, an electrode is firstly affixed between medial head and lateral head of gastrocnemius, and another is attached 3-5 cm distal from the first. It is important that electrodes be aligned along the long axis of the muscle for accurate measurement; values of the potential vary significantly if electrodes are placed across, at right angles or otherwise, in relation to the muscle's long axis.

Prevention of contamination from artifacts is also a major issue. Contamination of the potential induced by the latency of about $10 \mathrm{~ms}$ after the stimulus is highly likely specifically in terms of the inception and waveform of the $\mathrm{H}$-wave. To remove artifact contamination, it is important that electrodes be securely earthed and the width and size of square wave of electrical stimulation be closely monitored.

As for $\mathrm{H}$-wave amplitude, it increases as the motor neuron pool becomes more excitable due to weak voluntary muscle contraction, but $\mathrm{H}$-wave latency hardly changes. Based on this characteristic, those muscle groups from which $\mathrm{H}$-wave is hardly recorded at rest (e.g., tibialis anterior, extensor carpi radialis, and abductor pollicis brevis) can be induced to elicit recordable levels of $\mathrm{H}$-wave.

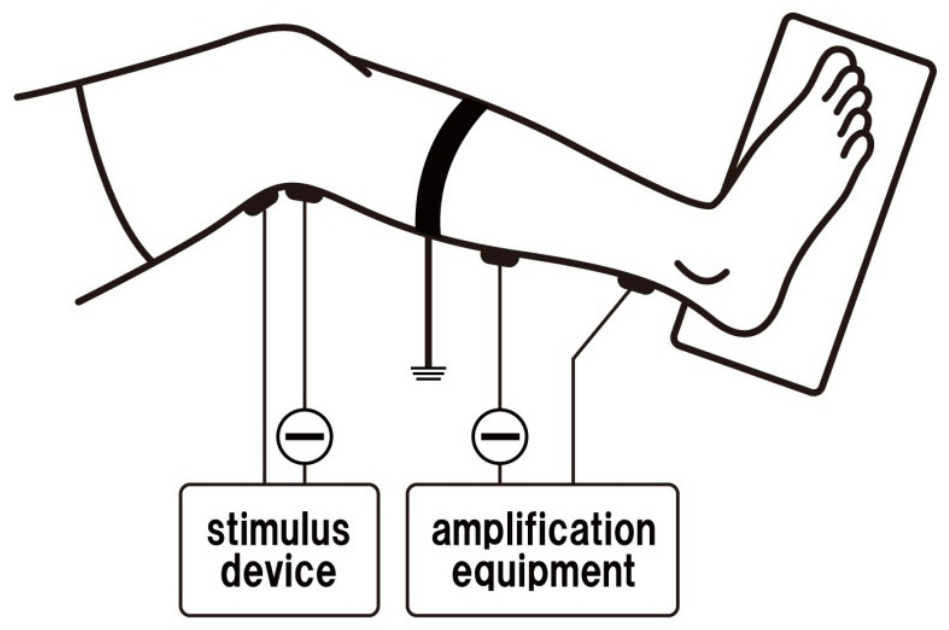

Figure 2. Recording method

\subsubsection{Identification of waveforms}

To elicit H-reflex from soleus muscle, a low-intensity electrical stimulation is applied on tibial nerve in the popliteal space, whereupon only those group Ia fibers with low threshold levels become selectively excited. The waveform which appears in the 20-30 ms latency is the $\mathrm{H}$-wave 
from soleus muscle. When the intensity of an electrical stimulus is gradually raised, group Ia fibers will be excited more. At the same time, alpha motor neuron which runs from the spinal cord to muscle fiber also becomes excited.

M-wave will appear at the 5-10 ms latency. If the stimulus intensity is raised, excitement of group Ia fibers will be lost and excitement of alpha motor neuron will become greater. $\mathrm{H}$-wave amplitude decreases, whereas M-wave amplitude increases. H-wave is lost before $\mathrm{M}$-wave reaches the maximum amplitude; only the M-wave may remain (Fig. 1).

$\mathrm{H}$-wave latency is measured at the time when the value shows the first deflection from baseline. In the soleus $\mathrm{H}$-wave, the first rising edge of the positive waveform is adopted as its latency because it is impossible to affix the recording electrode on the motor point. And its $\mathrm{H}$-wave amplitude is defined by the difference in potential either from baseline to the top of negative waveform, or from the top of negative waveform to the top of next positive waveform.

It is important to ensure that the same measurement method is used for $\mathrm{H}$-wave and $\mathrm{M}$-wave amplitude. If these two amplitudes were measured differently, the $\mathrm{H} / \mathrm{M}$ amplitude ratio would lose its reliability in the analysis of evoked EMG study.

\subsection{To evaluate the muscle tone of skeletal muscle by analysis of the $H / M$ ratio}

Under uniform stimulus conditions, the amplitude size of an H-wave is determined by the strength of the stimulus and the excitability of the reflex arc. Thus, $\mathrm{H}$-wave has been used as an indicator of the excitability of motor neurons in the anterior horn of the spinal cord.

In clinical practice, the ratio between the maximum amplitude of $\mathrm{H}$-wave (Hmax) and that of M-wave (Mmax), or H/M ratio, is often adopted as a good index. The Hmax is taken to reflect the number of excited alpha motor neurons in the anterior horn of the spinal cord, when the condition is adjusted so as to maximize the input from group Ia fibers upon electrical stimulation. The Mmax, on the other hand, is thought to show the amplitude of complex muscle action potential when all the alpha motor neurons dominating the muscle (soleus muscle here) are excited synchronously. That is, of all the alpha motor neurons that dominate the targeted muscle (e.g., soleus muscle), the $\mathrm{H} / \mathrm{M}$ ratio shows the percentage of excited alpha motor neurons upon electrical stimulation. In fact, correlation has been observed between the H/M ratio and the degree of spasticity. The $\mathrm{H} / \mathrm{M}$ ratio shows marked increases in the elevated excitability of alpha motor neurons in the spinal cord, or in patients with spasticity.

Conversely, in cases of peripheral neuropathy, this $\mathrm{H} / \mathrm{M}$ ratio is decreased. Because there is a great difference between individuals in the $\mathrm{H} / \mathrm{M}$ ratio, it is more useful as a therapeutic tool in the same patient rather than as a general diagnostic tool. The $\mathrm{H} / \mathrm{M}$ ratio would work when you tried to make an objective judgment of therapeutic effects on immediate change in the same patient, or when you tried to make a longitudinal change in a given condition also in the same patient.

It is surmised that if you observe changes in the H-wave at the time of intervention such as muscle contraction, vibration stimulation to the tendon, or muscle stretching, then those 
changes have in fact been brought on by the intervention, and not due to alterations in the physical condition such as electrode attachment and the like.

\section{Vibration stimuli}

\subsection{Responses of skeletal muscle to vibration stimulation}

Vibration stimulation has been used as one of the treatment modes for conditions of muscle tone of skeletal muscle, including spasticity. Its effects have in fact been reported. Unlike manual therapy, vibration stimulation is an excellent treatment method because it is expected to show a certain effect regardless of who uses it.

\subsubsection{Physiological act on muscle cells}

Though its detailed mechanism has not been elucidated, vibration stimulation acts directly on the contracted muscle cells to relax them (Vukas 1978, Ljung 1972, Ljung 1975).

\subsubsection{Inhibitory act on muscle contraction}

When skeletal muscle during a maximum contraction receives vibration stimulation, the muscle's action potential on EMG, nerve activity, and muscle output are reduced (Bongiovanni 1989, Kouzaki 2000, Stephen 2003, Konishi 2009). The inhibitory effect of muscle contraction is dependent on the conditions of vibration stimulation: the effect is greater if the frequency of the vibration stimulation is low, or if its amplitude is large (Desmedt 1978).

In recent reports, when stroke patients with a spastic upper limb received vibration stimulation on the limb, their upper limb function was shown to improve with a suppression of muscle tone (Noma 2012, Caliandro 2012).

In patients who underwent ACL reconstructive surgery or those with osteoarthritis, vibration stimulation to their quadriceps femoris muscle brought about neither an increase in neural activity as evaluated by integrated EMG nor a decrease in muscle output as evaluated by peak torque (Konishi 2002, Rice 2011). It is thought that this outcome was due to a failure in Ia afferent feedback, including dysfunction of the gamma-loop.

\subsubsection{Neurophysiological mechanism}

Muscle spindle senses a very small change in muscle length when a skeletal muscle receives vibration stimulation. And this information is transmitted to the fibers of group Ia or II, eventually to reach the spinal cord. In the spinal cord, the information serves as presynaptic inhibition through an interstitial cell and suppresses the alpha motor neuron (Gillies 1969, Shinohara 2005).

In addition, sustained vibration stimulation will selectively excite group Ia fibers. That will then bring about, in nerve endings, depletion of neurotransmitters and a rising threshold of 
group Ia fibers (Kouzaki 2000). It is believed that this is one of the reasons why a decrease is observed in the excitability of motor neurons in the spinal cord.

\subsubsection{Conditions for vibration stimulation}

The equipment that is used to apply vibration stimulation may vary from researcher to researcher, but the effect seems to be uniform as long as stimulation conditions are controlled in the same way. Stimulation conditions inevitably differ according to the research objective, but they are generally set at a frequency of $50-100 \mathrm{~Hz}$ and amplitude of $1-2 \mathrm{~mm}$. The equipment is contacted at $20-30 \mathrm{~N}$ on the body or tendon of the targeted skeletal muscle.

Effects that vibration stimulation has on the body depend on the duration of stimulation. The $\mathrm{H} / \mathrm{M}$ ratio decreases immediately after the application of stimulation, and the inhibitory effect on spasticity has been confirmed in 5 minutes of stimulation (Noma 2012). Muscle output is reduced by a sustained stimulation of at least 20 minutes (Bongiovanni 1989, Kouzaki 2000, Stephen 2003, Konishi 2009).

\subsection{Our previous study 1}

Vibration stimulation on a skeletal muscle of healthy adults is known to bring on effects such as reflexivity contraction of an agonist muscle by muscle spindle Ia afferent nerve excitability, and inhibition of the antagonist muscle, called "tonic vibration reflex". Vibration stimulation also causes inhibition of monosynaptic reflexes such as the tendon reflex and stretch reflex while stimulation with vibration continues. These effects vary depending on stimulus conditions.

Clarified issue 1: Anterior horn cells in the spinal cord are suppressed by vibration stimulation from its onset to about 3 minutes into stimulation.

As a result of vibration stimulation on the triceps surae of healthy adults, the H/M ratio continues to decrease from 1 to 3 minutes after stimulus onset. After 4 minutes into stimulation, though the ratio continues to decrease somewhat, no significant difference was found between values at after 4 minutes and the value at 3 minutes of stimulation.

From these findings, it has been clarified that excitability of alpha motor neurons gets suppressed immediately after the intervention with vibration stimulation. Also clarified was that the decline of the $\mathrm{H} / \mathrm{M}$ ratio reaches a steady state after 3 minutes of stimulation from its onset.

\section{Clarified issue 2: It is recommended that vibration stimulation be applied on tendon.}

If vibration stimulation is to be adopted in clinical settings, it is essential that an appropriate set of stimulus conditions should be considered for each of the clinically different cases. A site of stimulation is certainly one of the factors for the treating therapist to take into account.

Based on the results of our study, the $\mathrm{H} / \mathrm{M}$ ratio was significantly lower after the onset of vibration stimulation on both muscle belly and the tendon $(\mathrm{p}<0.01)$. In addition, when stimulus to the belly was compared with stimulus to the tendon, the $\mathrm{H} / \mathrm{M}$ ratio was significantly lower in the latter $(\mathrm{p}<0.05)$. In other words, to suppress muscle tone using a vibration stimulus, it is recommended that a stimulus be applied on the tendon. 


\subsection{Our previous study 2}

When vibration stimulation is applied on skeletal muscle, the amount of output and muscle activity of the stimulated skeletal muscle is decreased. This finding should help to bring on a change in the pattern of muscle activity during action (Table 1).

It may be possible to facilitate the activity of the muscle that is important to knee joint extension, namely vastus lateralis muscle, when vibration stimulation decreases its activity. In fact, we have confirmed that, when vastus lateralis muscle was stimulated by vibration, the reduction in the amount of muscle activity was observed in only vastus lateralis muscle among quadriceps femoris muscles (Table 2).

There may be a possibility of enhancing the activity of inner muscles, which were long assumed to be unsusceptible to strengthening, provided that you can selectively suppress just those muscles you want to suppress. It is hoped that our future studies will clarify this issue as well.

\begin{tabular}{cc}
\hline Pre-vibration & Post-vibration \\
\hline $250.0 \pm 25.4$ & $220.5 \pm 16.2$ \\
\hline
\end{tabular}

Mean \pm standard deviation

Table 1. Muscle force of quadriceps femoris muscle before and after vibration stimulation (\%BW).

\begin{tabular}{lll}
\hline & Pre-vibration & Post-vibration \\
\hline Rectus femoris muscle & $174.5 \pm 29.2$ & $121.2 \pm 52.3$ \\
\hline Vastus medialis muscle & $143.8 \pm 34.2$ & $102.1 \pm 68.5$ \\
\hline Vastus lateralis muscle & $113.6 \pm 49.1$ & $95.5 \pm 37.8 *$ \\
\hline${ }^{*} \mathrm{p}<0.05:$ pre-vibration vs post-vibration & \\
Mean \pm standard deviation & & \\
\hline
\end{tabular}

Table 2. Muscle activity of quadriceps femoris muscle before and after vibration stimulation (\%iEMG).

\subsection{Previous studies by other authors}

\subsubsection{Spasticity of upper limb in stroke patients is suppressed by 5 to 10 minutes of vibration stimulation}

If vibration stimulation of about 5 to 10 minutes is applied on the spastic muscle of a stroke patient's upper limb, the following will be observed: a decrease in the H/M ratio indicative of the excitability level of anterior horn cells in the spinal cord; improvement of motor function and of Modified Ashworth Scale indicative of the degree of spasticity; and improvement of Functional Ability Scale using Wolf Motor Function Test. In some treatment cases, an immediate effect was recorded after the intervention.

The nervous system of a spastic stroke patient may be restructured and/or strengthened by adjusting the level of CNS excitability using a vibration stimulus in combination with training 
on the nervous system. It is anticipated that further effects that vibration stimulation has on spastic muscle in stroke patients will be revealed in the near future.

2.4.2. Muscle activity and the amount of skeletal muscle output are reduced by more than 20 minutes of vibration stimulation

Vibration stimulation on a sustained maximum muscle contraction case can reduce the firing frequency of the nerve cells involved in the maximum contraction, and subsequently the muscle output will decrease. In addition, more than 20 minutes of vibration stimulation on quadriceps femoris muscle can bring on a reduction in the peak torque and integrated EMG.

If you apply these findings clinically, it is possible to suppress the excessive and abnormal muscle activity that occurs as a compensatory motion during action, and subsequently to let the patient have efficient training.

However, in patients with osteoarthritis of the knee and also in patients who have undergone ACL reconstruction and therefore need onerous training, no decrease of the peak torque and integrated EMG occurs after the vibration stimulation. Abnormal gamma motor neurons are believed to be involved, and the abnormality results from reduced afferent nerve activity of the joints.

All in all, clinical application of vibration stimulation on patients with musculoskeletal disorders awaits further trials because there remain some unsettled issues.

\section{Inhibitory and facilitative effects of muscle tone in normal healthy subjects}

This section provides an overview of the effects of inhibition and facilitation by physical stimuli on skeletal muscle of healthy subjects. We shall then consider the current state surrounding the physical stimulus in the field.

\subsection{Pressure stimulation}

A decreased $\mathrm{H} / \mathrm{M}$ ratio was observed by air pressure in the splint on triceps surae muscle of the lower limb (Robichaud et al., 1992) and radiocarpal flexor muscle of the upper limb (Agostinucci et al., 2006) both in healthy subjects.

Pressure stimulation causes a decrease in blood flow, leads to the state of lack of oxygen, and adversely affects the site of compression of the cell, tissue, or organ. What then would be an appropriate duration and compression strength to apply without bringing on undesirable effects? In a study of healthy Japanese youths, the condition of 5 minutes at $50 \mathrm{mmHg}$ was recommended as appropriate (Miura et al., 2011). A pressure of up to $50 \mathrm{mmHg}$ did not result in any statistically significant differences in blood flow compared with no-addition pressure stimuli. But at this time the excitability of soleus muscle motor neurons was inhibited in the spinal cord. 


\subsection{Whole Body Vibration (WBV)}

Studies using WBV include the examination of its longitudinal and acute effects. Indices that are adopted to verify or refute those effects are as follows: muscle activity, muscle strength, power, height by counter jump, body balance, and mechanical competence of bones.

\subsubsection{Acute effects}

Neuromuscular stimulation derived from WBV is the likely source of previously observed changes in athletic performance. The tonic vibration reflex is a response elicited from vibration directly applied to a muscle belly or tendon. This reflex is characterized by activation of muscle spindles primarily though Ia afferents and activation of extrafusal muscle fibers through alpha motor neurons (Cormie et al., 2006).

Vibration has been shown to stimulate transient increases in certain hormones, such as growth hormone and IGF-I (Cormie et al., 2006).

A study exists in which the flexibility of hamstring muscle of the lower limb was examined by varying the frequency of vibration stimulation. It showed that there was a $10 \%$ increase in flexibility at $20 \mathrm{~Hz}$ and no change at $40 \mathrm{~Hz}$ (Cardinale et al., 2003).

Vibration is detected not only by spindle, but also by the skin, the joint and secondary endings. All those structures contribute to the facilitatory input to the gamma-system which in turn affects sensitivity of the primary endings. Modulation of neuromuscular response to vibration is then to be referred not just to spindle activation, but to all the sensory systems in the body. Various parameters can affect the synergies in the sensory system and determine specific responses. Vibration is thought mainly to inhibit the contraction on antagonist muscles via Ia inhibitory neurons. However there is some evidence as well that vibrations can also produce co-activation (Cardinale et al., 2003).

A previous study investigated the acute effects of WBV on back and abdominal muscle activity. Muscle activity with vibration showed a low to moderate increase in trunk muscle activation (Wirth et al., 2011).

\subsubsection{Long-term effects}

A 4-month WBV-loading induced a significant $8.5 \%$ mean increase in jump height of young healthy adults. This improvement was already seen after 2 months of the vibration. Lower limb extension strength was also enhanced by the 2-month vibration period. Intervention with a 4-month WBV enhanced jumping power in young adults, suggesting neuromuscular adaptation to the vibration stimulation. On the other hand, the vibration-intervention showed no effect on dynamic or static balance of the subjects (Torvinen et al., 2002).

\subsubsection{The difference between athletes and normal healthy subjects}

There are many reports in which knee extensor strength and jump performance improved after stimulation with WBV in healthy subjects. In contrast, no improvement was observed in sprint- 
trained athletes and elite female field hockey players. This means either that the effect of daily training was greater than the stimulatory effect with WBV or that its cumulative activity and inhibitory effects were retained in the body system. It is suggested that the stimulating effect of WBV is probably greater in subjects that do not exercise every day.

\section{Inhibitory and facilitative effects of muscle tone in patients with pathological disorder}

Section 4 presents an overview of the effects of inhibition and facilitation provided by the physical stimulus on skeletal muscles in people with pathological disorder. We shall then consider the existing state surrounding the equipment used in rehabilitation.

In the field of rehabilitation, people with muscle hypo-tone are induced to undergo activities that facilitate muscle activity, and those with muscle hyper-tone are given activities that inhibit muscle activity. In general, when standing position is sustained there will be increases in muscle tone from that posture. Therapists often suppress the excessive muscle activity in such cases. Muscle hypo-tone from paralysis or hemiplegia is usually treated with a facilitative method using electrical stimulation or proprioceptive neuromuscular stimulation. At the present time, it is not clear to what extent these physical means are effective; it needs continued investigation. Physical therapy stands for intervention with physical means in people with physical disturbance so that their physical disorder is alleviated. For that purpose, application of clinical and kinesiologic EMG is one of the most effective methods.

\subsection{In people with cerebrovascular accident}

Cerebrovascular Accident (CVA) is a general term for diseases such as subarachnoid hemorrhage, rupture of a cerebral aneurysm, and cerebral infarction. Disturbances after a CVA are various in severities. What was the onset of a CVA like, was there early treatment, or was the site known at the time of its onset? These are the questions that help determine how severe CVAs turn out to be. Sequelae such as motor paralysis, sensory disturbances, and language disorder occur in about one-third of the patients who suffered CVA.

\subsubsection{Electrical stimulation}

When the antagonist muscle of spastic paralysis undergoes electrical stimulation, its muscle spasticity is suppressed. This suppression is brought on by reciprocity through the Ia inhibition in the spinal cord, which in turn results from an adjustment of the reflective circuit. If the spastic muscle itself is subjected to electrical stimulation in such a case, a decrease in muscle tone occurs through the antagonist inhibition. Upon application of electrical stimulation to rectus femoris muscle of the lower limb, facilitation of the flexion of the hip joint and the extension of the knee joint occurs through suppression of the muscle tone of hip flexor muscle and knee extensor muscle, respectively. Thus, gait itself turns out to show improvement. 
If a muscle receives electrical stimulation, what will be the reaction of the muscle? Electrical stimulation to a paralyzed muscle, for instance, will cause the amount of muscle activity to increase. This is referred to as the carry over effect.

\subsubsection{Pressure stimulation}

Pressure stimulation on spastic muscle of people with CVA brings about an effect that is similar to the effect it has on healthy people. Air pressure in a splint to triceps surae muscle of the lower limb in people with spastic paralysis caused a decrease in the H/M ratio (Robichaud et al., 1992). And the same was seen in spastic muscle of the upper limb: radiocarpal flexor muscle in people with neurological diseases, when stimulated by air pressure in the splint, registered a lowered H/M ratio (Agostinucci et al., 2006).

\subsection{In people with spinal cord injury}

Spinal cord injury (SCI) is a disorder caused by damage to the spinal nerve running through the spinal canal. SCI often brings on motor paralysis or sensory disturbances caused by their proprioceptive CNS diseases, leading eventually to impaired autonomic nervous system. Hypertensive tendon reflexes are often seen in the patient.

\subsubsection{Pressure stimulation}

When air pressure in a splint is applied to soleus muscle of the lower limb in people with SCI, alpha motor neuron reflex excitability is suppressed (Robichaud et al., 1992).

\subsubsection{Therapeutic massage}

When people with SCI were given massage treatment for 3 minutes, there was a decrease in the amplitude of H-reflex when compared with what it was before the massage (Goldberg et al., 1994).

\subsubsection{Whole Body Vibration (WBV)}

When WBV is applied on a person with SCI, residual effects of medication for muscle spasticity will sometimes be recognized. And because the duration of stimulation differs among researchers, a simple comparison is difficult (Ness et al., 2009).

There is a report in which the adopted cycle of WBV treatment consists of the following: 4 bouts of 45 seconds with one minute of seated rest between bouts, done on 3 days a week lasting for 4 weeks.

\subsection{In people with Parkinson's disease}

Parkinson's disease (PD), often caused by depletion of dopamine in the substantia nigra of the midbrain, is a disease that indicates a variety of movement disorders. Rigidity, tremor, 
akinesia, amimia, and pill-rolling phenomenon are the characteristic symptoms. By taking Ldopa, people with PD can alleviate these symptoms.

\subsubsection{Whole Body Vibration (WBV)}

\subsubsection{Acute effects}

With WBV, scores of Unified Parkinson's Disease Rating Scale (UPDRS) tremor and rigidity improved compared with no intervention. There is no evidence, however, that WBV is effective in improving knee proprioception and other clinical measures of sensorimotor performance, such as balance and mobility (Lau et al., 2011).

\subsubsection{Long-term effects}

WBV had no significant effects, or was only slightly effective, on UPDRS motor scores (Lau et al., 2011).

\subsection{In people with multiple sclerosis}

Multiple sclerosis (MS) is a chronic inflammatory, demyelinating disease of the central nervous system. The most prevalent symptoms of this disease include sensory changes, visual disturbances, fatigue, and micturition disorders (Jackson et al., 2008).

\subsubsection{Whole Body Vibration (WBV)}

\subsubsection{Acute effects}

There is no evidence that WBV is effective in improving peak torque values for both quadriceps and hamstring muscles (Jackson et al., 2008).

\subsubsection{Long-term effects}

Under the duration of 5 training sessions per 2-week cycle for 20 weeks, WBV did not improve leg muscle performance or functional capacity in mildly to moderately impaired people with MS (Broekmans et al., 2010). Also 8-week exercise is effective in improving standing balance and timed up-and-go test (Mason et al., 2012). But there are differences in stimulation frequency and duration between the two reports. That makes it difficult to determine whether WBV is or is not effective in the long run.

\subsection{In people with knee osteoarthritis}

\subsubsection{Whole Body Vibration (WBV)}

Previous research investigated WBV as an alternative strengthening regimen in the rehabilitation of people with total knee arthroplasty (TKA) compared with traditional progressive 
resistance exercise (TPRE). Post-TKA subjects received physical therapy with WBV or with TPRE for 4 weeks. There was a significant increase in knee extensor strength and improvements in mobility both with WBV and with TPRE.

With an addition of WBV to squat training, elderly people with knee osteoarthritis (OA) were evaluated on functional performance and self-report of disease. A total of 23 elderly subjects participated. There was no statistically significant difference in functional performance and self-report of disease status between groups of squat training with or without WBV.

\subsection{Summary}

We therapists realize after all that, at the present time, WBV effectiveness against SCI, PD, MS, and knee OA has not been established. There are reasons for that: disease mechanisms have not been clarified, and optimum conditions such as stimulation frequency are not definitely set yet. And in intervention studies, one cannot completely eliminate the influence of other factors. It is unfortunate that not enough high-level evidence-based research has been done or published.

Though rather limited in scope, we therapists can help people with disabilities, or even people without disabilities, by suppressing the muscle tone of a given muscle through the spinal cord loop.

\section{Health science research with EMG}

In research using EMG, there are two groups of studies: clinical and kinesiologic. Kinesiologic EMG is to record the response during motion of the body.

\section{Clinical EMG}

1. EMG as a diagnostic aid in cases in which causes of muscle weakness and muscle atrophy are neurogenic or myogenic

2. EMG as a means to confirm the lesion site and properties of peripheral nerve injury

3. EMG as a means to confirm abnormal muscle activity

4. EMG in cases that are not classifiable as 1,2, or 3 above

Likely target diseases are amyotrophic lateral sclerosis, polymyositis, carpal tunnel syndrome, and Bell's palsy.

Cases of kinesiologic EMG are as in the following:

1. EMG to check presence or absence of muscle activity

2. EMG to measure timing and duration of muscle activity

3. EMG to measure amount of muscle activity 
4. EMG as basis of biofeedback to patients

5. EMG to determine matters relative to muscle fatigue

6. EMG to measure reaction time on external stimuli

7. EMG to evaluate motor control

8. EMG to assess gait

9. EMG to evaluate motor learning

10. EMG for purposes not classifiable as 1 to 9 above.

In recent years, the study of human-machine interfaces became fairly popular. For example, equipment is used to amplify muscle activities of a person with disabilities, which will then be relayed to a robot so it can put them into effect for the disabled person. Or EMG is made use of as a vital part of technique to convey information from a robot to a human subject through the human senses. We shall introduce some of these studies.

In the field of rehabilitation, the type of equipment has been developed that controls movements of a joint by the muscle discharge in people with disabilities. The equipment sends a command from electrodes attached to the forearm of the robot to its artificially created fingers to control. The number of sensors attached on the disabled person increased gradually, so it became possible to control the intensity of such delicate movements as tapping or lifting. Numerous researches have also been done on motion control by an exoskeleton. The target joints now include forearm, elbow joint, shoulder joint, upper limb, cervix, hip joint, knee joint and lower limb, as well as a wide variety of fingers and hands. In upper limb research, starting from motions of three degrees of freedom (3-DOF), it has now reached the level of 7-DOF motions. Research on reaction time has also shown improvement; in the line of research on hand reactions, delays of only $100 \mathrm{~ms}$ have been attained. Research has also been done on robot-assisted therapy on upper limb recovery after stroke, but not much that is revolutionary has come out of it so far. Perhaps we can expect to see this line of research grow in the future.

We consider it possible that robot-assisted therapy may eventually help an individual patient become free from environmental and personal factors. Introduction of the so-called ICF (International Classification of Functioning, Disability and Health) model may be something that is revolutionary.

\section{Conclusion}

Verification of inhibitory effects of muscle tone by vibration stimulation as we did above, though it is only the beginning, seems to point to further development in the future. Significance may be found in the fact that healthy people can be checked using simple equipment. In the literature, a certain level of effectiveness of vibration stimulation is found in people with disabilities; determination of the optimum stimulation conditions will certainly be researchers' next point of interest. In studies using other physical means, it seems necessary to set conditions 
for measuring the effectiveness of the given means. Overall, studies of equipment-aided therapies tell us that aided-therapies seem to be superior to non-aided therapies because you can minimize the individual difference between techniques of intervention. This may be true in day-to-day therapeutic sessions as well as in the examination of the effect of physical therapy.

\section{Acknowledgements}

The authors are grateful to Mr. Kensuke Tokaichi for refining the English of the manuscript.

\section{Author details}

Satoru Kai ${ }^{1}$ and Koji Nakabayashi ${ }^{2}$

1 Faculty of Allied Health Sciences, Kansai University of Welfare Sciences, Japan

2 Department of Physical Therapy, Takeo Nursing and Rehabilitation School, Japan

\section{References}

[1] Agostinucci, J, Holmberg, A, Mushen, M, Plisko, J, \& Gofman, M. (2006). The effects of circumferential air-splint pressure on flexor carpi radialis H-reflex in subjects without neurological deficits, Percept. Mot. Skills, , 103, 565-579.

[2] Bongiovanni, L. G, Hagbarth, K. E, \& Stjernberg, L. (1989). Prolonged muscle vibration reducing motor output in maximal voluntary contractions in man, J. Physiol, $423,15-26$.

[3] Broekmans, T, Roelants, M, Alders, G, Feys, P, Thijs, F, \& Eijnde, B. O. (2010). Exploring the effects of a 20-week whole-body vibration training programme on leg muscle performance and function in persons with multiple sclerosis, J. Rehabil. Med , 42, 866-872.

[4] Caliandro, P, Celletti, C, Padua, L, Minciotti, I, Russo, G, Granata, G, La Torre, G, Granieri, E, \& Camerota, F. (2012). Focal muscle vibration in the treatment of upper limb spasticity: A pilot randomized controlled trial in patients with chronic stroke, Arch. Phys. Med. Rehabil, Apr 13.

[5] Cardinale, M, \& Lim, J. (2003). The acute effects of two different whole body vibration frequencies on vertical jump performance, Med. Sport , 56, 287-292. 
[6] Cormie, P, Deane, R. S, Triplett, N. T, \& Mcbride, J. M. (2006). Acute effects of wholebody vibration on muscle activity, strength, and power, J. Strength Cond. Res, 20, 257-261.

[7] Desmedt, J. E, \& Godaux, E. (1978). Mechanism of the vibration paradox: excitatory and inhibitory effects of tendon vibration on single soleus muscle motor units in man, J. Physiol , 285, 197-207.

[8] Gillies, J. D, Lance, J. W, Neilson, P. D, \& Tassinari, C. A. (1969). Presynaptic inhibition of the monosynaptic reflex by vibration, J. Physiol , 205, 329-339.

[9] Goldberg, J, Seaborne, D. E, Sullivan, S. J, \& Leduc, B. E. (1994). The effect of therapeutic massage on H-reflex amplitude in persons with a spinal cord injury, Phys. Ther , 74, 728-737.

[10] Jackson, S. W, \& Turner, D. L. (2003). Prolonged muscle vibration reduces maximal voluntary knee extension performance in both the ipsilateral and the contralateral limb in man, Eur. J. Appl. Physiol , 88, 380-386.

[11] Jackson, K. J, Merriman, H. L, Vanderburgh, P. M, \& Brahler, C. J. (2008). Acute effects of whole-body vibration on lower extremity muscle performance in persons with multiple sclerosis, JNPT, , 32, 171-176.

[12] Kaeser, H. E. (1973). Evoked muscle and sensory nerve action potentials in polyneuropathies. In: Handbook of Electroencephalography and Clinical Neurophysiology, Elsevier, Amsterdam, , 16A, 36-44.

[13] Konishi, Y, Fukubayashi, T, \& Takeshita, D. (2002). Possible mechanism of quadriceps femoris weakness in patients with ruptured anterior cruciate ligament, Med. Sci. Sports Exerc , 34, 1414-1418.

[14] Konishi, Y, Kubo, J, \& Fukudome, A. (2009). Effects of prolonged tendon vibration stimulation on eccentric and concentric maximal torque and EMGs of the knee extensors, Journal of Sports Science and Medicine, , 8, 548-552.

[15] Kouzaki, M, Shinohara, M, \& Fukunaga, T. (2000). Decrease in maximal voluntary contraction by tonic vibration applied to a single synergist muscle in humans, J. Appl. Physiol , 89, 1420-1424.

[16] Lau, R. W. K, Teo, T, Yu, F, Chung, R. C. K, \& Pang, M. Y. C. (2011). Effects of wholebody vibration on sensorimotor performance in people with Parkinson disease: A systematic review, Phys. Ther, 91, 198-209.

[17] Ljung, B, \& Sivertsson, R. (1972). The inhibitory effect of vibrations on tension development in vascular smooth muscle, Acta. Physiol. Scand , 85, 428-430.

[18] Ljung, B, \& Hallgren, P. (1975). On the mechanism of inhibitory action vibrations as studied in a molluscan catch muscle and in vertebrate vascular smooth muscle, Acta. Physiol. Scand, 95, 424-430. 
[19] Mason, R. R, Cochrane, D. J, Denny, G. J, Firth, E. C, \& Stannard, S. R. (2012). Is 8 weeks of side-alternating whole-body vibration a safe and acceptable modality to improve functional performance in multiple sclerosis?, Disabil. Rehabil , 34, 647-654.

[20] Miura, N, Kurosawa, K, Hirose, M, \& Suzuki, T. (2011). Inhibitory effects of pressure on soleus muscle motor neuron excitability and flow volume in healthy adults, Rigakuryoho Kagaku, , 26, 773-776.

[21] Nakabayashi, K, Kodama, T, Mizuno, K, Ikeda, T, Kai, N, Fukura, T, \& Kai, S. (2011). The inhibitory effect of vibration stimulus on muscle tone of the triceps surae: Analysis of the H/M ratio, Rigakuryoho Kagaku, , 26, 393-396.

[22] Nakabayashi, K, Kodama, T, Matsumoto, N, Yamamoto, H, Nomiyama, M, Fukura, T, \& Kai, S. (2012). Muscle tone inhibitory effects of vibration applied to the tendon and muscle belly of the triceps surae, Rigakuryoho Kagaku, , 27, 151-154.

[23] Ness, L. L, \& Field-fote, E. C. (2009). Effect of whole-body vibration on quadriceps spasticity in individuals with spastic hypertonia due to spinal cord injury, Restor. Neurol. Neurosci, 27, 623-633.

[24] Noma, T, Matsumoto, S, Shimodozono, M, Etoh, S, \& Kawahira, K. (2012). Anti-spastic effects of the direct application of vibratory stimuli to the spastic muscles of hemiplegic limbs in post-stroke patients: a proof-of-principle study, J. Rehabil. Med, 44, 325-330.

[25] Rice, D. A, Mcnair, P. J, \& Lewis, G. N. (2011). Mechanisms of quadriceps muscle weakness in knee joint osteoarthritis: the effects of prolonged vibration on torque and muscle activation in osteoarthritic and healthy control subjects, Arthritis Res. Ther R151, 13

[26] Robichaud, J. A, Agostinucci, J, \& Linden, D. W. V. (1992). Effect of air-splint application on soleus muscle motoneuron reflex excitability in nondisabled subjects and subjects with cerebrovascular accidents, Phys. Ther, 72, 176-183.

[27] Shinohara, M, Moritz, C. T, Pascoe, M. A, \& Enoka, R. M. (2005). Prolonged muscle vibration increases stretch reflex amplitude, motor unit discharge rate, and force fluctuations in a hand muscle, J. Appl. Physiol , 99, 1835-1842.

[28] Torvinen, S, Kannus, P, Sievanen, H, Jarvinen, T. A. H, Pasanen, M, Kontulainen, S, Jarvinen, T. L. N, Jarvinen, M, Oja, P, \& Vuori, I. (2002). Effect of four-month vertical whole body vibration on performance and balance, Med. Sci. Sports Exerc, 34, 1523-1528.

[29] Vukas, M, Sivertsson, R, \& Ljung, B. (1978). Inhibitory effects of vibrations on contractility of isolated rabbit papillary muscle, Scand. J. Clin. Lab. Invest, 38, 415-419.

[30] Wirth, B, Zurfluh, S, \& Muller, R. (2011). Acute effects of whole-body vibration on trunk muscles in young healthy adults, J. Electromyogr. Kinesiol , 21, 450-457. 